\title{
CONCERNING SEMICONNECTED MAPS
}

PAUL E. LONG

Introduction. Professor John Jones, Jr., [3], introduces a semiconnected map $f: X \rightarrow Y$ as one in which $f^{-1}$ preserves closed connected subsets of $Y$, and gives conditions under which a semiconnected map is continuous or is a homeomorphism. Theorem 1 of that paper is generalized here, and comparisons are made between semiconnected maps and other noncontinuous maps.

Among the several other well-known types of noncontinuous maps only the connected map and the connectivity map will be considered. A connected map $f: X \rightarrow Y$ is one which preserves connected subsets of $X$ and a connectivity map $f: X \rightarrow Y$ is one for which the induced graph map, $g: X \rightarrow X \times Y$ defined by $g(x)=(x, f(x))$ for each $x \in X$, is connected. It is easy to see that if $f: X \rightarrow Y$ is continuous, then $f$ is a connectivity map, and if a connectivity map, then also connected. Examples showing the reverse implications are not always valid may be found in [2]. The example $f(x)=x^{2}$ from the reals into the reals (usual topology in both cases) shows that continuous maps, hence connected and connectivity maps, need not be semiconnected. Furthermore, $f(x)=x$ from the reals (usual topology) to the reals (discrete topology) is semiconnected but not connected, hence not a connectivity nor a continuous map.

Throughout, $\operatorname{cl}(A)$ denotes the closure of the set $A$.

Results. Theorem 1 generalizes Theorem 1 of [3].

Theorem 1. If $f: X \rightarrow Y$ is semiconnected and onto the semi-locallyconnected space $Y$, then $f$ is continuous.

Proof. Let $B \subset Y$ be open. It will be shown that $f^{-1}(B)$ is open in $X$. For each point $b \in B$ there exists an open set $V_{b} \subset B$ such that $Y-V_{b}$ consists of a finite number of components $C_{1}^{b}, C_{2}^{b}, \cdots, C_{n}^{b}$. Each $C_{j}^{b}$ is closed and connected; hence $f^{-1}\left(C_{j}^{b}\right)$ is closed and connected since $f$ is semiconnected. Thus $U_{j-1}^{n} f^{-1}\left(C_{j}^{b}\right)$ is closed and contains no point of $f^{-1}\left(V_{b}\right)$ so that $X-\cup_{j=1}^{n} f^{-1}\left(C_{j}^{b}\right)=R_{b}$ is an open set in $X$ having the property that $f\left(R_{b}\right)=V_{b}$. Consequently $\bigcup_{b \in B} R_{b}$ is open in $X$ and furthermore $f^{-1}(B)=\bigcup_{b \in B} R_{b}$.

TheOREM 2. Let $f: X \rightarrow Y$ be a closed map where $f^{-1}(y)$ is connected for each $y \in Y$. Then if $M \subset Y$ is connected, $f^{-1}(M)$ is connected.

Received by the editors April 15, 1968. 
Proof. Considering $M \subset Y$ nondegenerate, suppose $f^{-1}(M)$ $=H \cup K$, separated. Then $f(H) \cup f(K)=M$ and one of the sets, say $f(H)$, has a limit point $y_{0}$ of the other, $f(K)$ in this case. Since $f^{-1}(y)$ is connected for each $y \in Y, f^{-1}\left(y_{0}\right) \subset H$ and furthermore $f(H) \cap f(K)$ $=\varnothing$. Consequently, because $\operatorname{cl}(K) \cap H=\varnothing, y_{0} \notin f(\operatorname{cl}(K))$ which contradicts $f$ being closed. The conclusion that $f^{-1}(M)$ is connected follows.

Corollary 1. Let $f: X \rightarrow Y$ be a closed semiconnected map where $Y$ is $T_{1}$. Then if $M \subset Y$ is any connected set, $f^{-1}(M)$ is connected.

Corollary 2. Let $f: X \rightarrow Y$ be a closed connected map where $f^{-1}(y)$ is connected for each $y \in Y$ and $Y$ is $T_{1}$. Then $f$ is semiconnected.

Proof. For any closed connected $M \subset Y, f^{-1}(M)$ is connected by Theorem 2. By $[4] f^{-1}(M)$ is also closed and hence $f$ is semiconnected.

Corollary 3. If $f: X \rightarrow Y$ is a closed continuous map where $f^{-1}(y)$ is connected for each $y \in Y$, and $Y$ is $T_{1}$, then $f$ is semiconnected.

THEOREM 3. Let $f: X \rightarrow Y$ be continuous where $f^{-1}(y)$ is connected for each $y \in Y, X$ is countably compact first countable and $Y$ is $T_{1}$ first countable. Then $f$ is semiconnected.

Proof. Let $M \subset Y$ be closed and connected. Continuity of $f$ insures $f^{-1}(M)$ closed. It will now be shown that $f^{-1}(M)$ is connected from which the conclusion that $f$ is semiconnected follows.

Suppose $f^{-1}(M)=H \cup K$, separated. Then $f(H) \cup f(K)=M$ and one of these sets, say $f(H)$, has a limit point $y_{0}$ of the other, $f(K)$ in this instance. There exists a sequence of distinct points $y_{n} \in f(K)$ such that $y_{n} \rightarrow y_{0}$ where $f^{-1}\left(y_{0}\right) \subset H$ and $f^{-1}\left(y_{n}\right) \subset K$ for each $n$. Extracting $x_{n} \in K \cap f^{-1}\left(y_{n}\right)$ for each $n$, the set $\left\{x_{n}\right\}$ has a limit point $x_{0} \notin H$ since $\operatorname{cl}(K) \cap H=\varnothing$. Thus $f\left(x_{0}\right) \neq y_{0}$. Since $X$ is first countable, there is a subsequence $\left\{x_{n_{k}}\right\}$ of $\left\{x_{n}\right\}$ such that $x_{n_{k}} \rightarrow x_{0}$. But $f\left(x_{n_{k}}\right) \rightarrow y_{0} \neq f\left(x_{0}\right)$ contradicting continuity of $f\left[1\right.$, Theorem 3.15, p. 102]. Thus $f^{-1}(M)$ is connected.

\section{REFERENCES}

1. D. W. Hall and G. L. Spencer II, Elementary topology, Wiley, New York, 1957.

2. O. H. Hamilton, Fixed points for certain non-continuous transformations, Proc. Amer. Math. Soc. 8 (1957), 750-756.

3. John Jones, Jr., On semiconnected mappings of topological spaces, Proc. Amer. Math. Soc. 19 (1968), 174-175.

4. D. E. Sanderson, Noncontinuous functions which act like continuous functions for connected sets, Notices Amer. Math. Soc. 3 (1964), 765.

University of ARKaNSAS 